\title{
Compact Optical Sensor for the Measurement of Surface Microtopographies on Turbine Blades
}

\author{
Yinan Li $^{1}$, Markus Kästner ${ }^{1}$ and Eduard Reithmeier ${ }^{1}$ \\ ${ }^{1}$ Institute of Measurement and Automatic Control, Gottfried Wilhelm Leibniz Universität Hannover, \\ Nienburger Str. 17, 30167 Hannover, Germany \\ Yinan.li@imr.uni-hannover.de
}

\begin{abstract}
:
The importance of measuring surface microtopographies for quality control has increased in the last decades. The inspection and measurement of complex shaped work pieces, like turbine blades or blisks (blade integrated disks), leads to a new challenge in measurement technology. In order to allow a precise measurement of surface microstructures, optical microscopes, such as confocal laser scanning microscopes and optical scanning microscopic interferometers, are usually used [1-3]. However, with common optical microscopes with 20x or 50x magnification objectives it is difficult to obtain a large spatial range which is necessary for measuring curved surfaces of turbine blades.

In this paper we present a compact non-contact measurement system based on a low coherence Michelson interferometer ( $\mathrm{LCl}$ ), which allows a nanometer-level vertical resolution and a millimeterlevel lateral range. The developed LCl-system using a telecentric lens allows a high accuracy surface microtopography measurement and a large FOV for measuring curved surfaces, which cannot be measured by common interferometric microscopes.
\end{abstract}

Key words: surface microtopography measurement, non-contact measurement system, lowcoherence interferometer ( $\mathrm{LCl})$.

\section{Introduction}

The measurement of the surface microtopography on complex durable products, like turbine blades, is a relevant prerequisite for the optimization of the aerodynamic performance [4]. For example, a laminar boundary layer on the blade surface with a low Reynolds number can cause a low skin friction [5]. Because turbine blades always have complex shapes and curved surfaces, it is difficult with a common interferometric microscope to obtain a large spatial range. For this reason we developed a compact noncontact measurement system with a long working distance that is able to measure the surface microtopography with nanometer-level vertical resolution and a millimeter-level lateral range. On the basis of a low coherence Michelson interferometer (LCl), with an optimized small sensor head, the system is perfectly qualified to provide the measurement of precision surfaces on turbine blades or a blisk (Blade integrated disk). Fig. 1 shows a virtual measurement example to demonstrate that the sensor head of the system is suitable for a small space between two blades on compressor blisks.

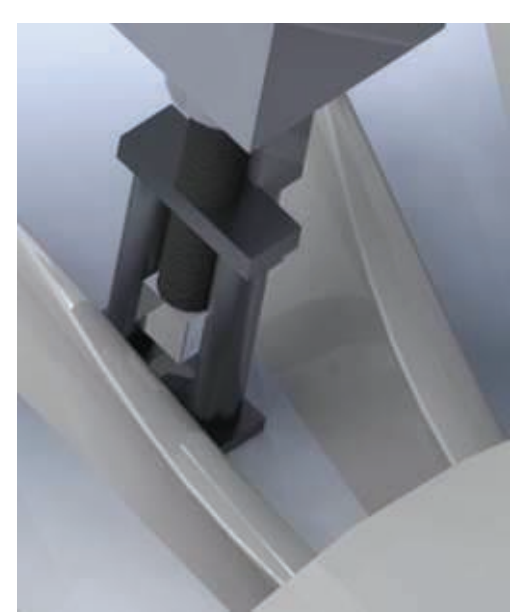

Fig.1. A virtual measurement example: surface measurement between two blades on a compressor blisk.

\section{Setup of the Measurement System}

In order to realize a precise surface measurement with a small sensor head, fig. 2 shows the schematic optical design for the experimental setup of the LCl-system. It consists of a low-coherence deep red lightemitting diode (LED) with a mean wavelength of $645 \mathrm{~nm}$ and a spectral bandwidth of $25 \mathrm{~nm}$ as light source, a charge coupled device (CCD) 
camera (model: Pike F-145B; cell size: 6.45 $\mu \mathrm{m}$; Resolution: 1388 x 1038 pixels), a $4 \mathrm{x}$ magnification telecentric lens with a wide depth of field (DOF) and a large field of view (FOV), a reference mirror driven by a precision piezo stage with two adjustable polarizers and a rotatable mirror at the sensor head.

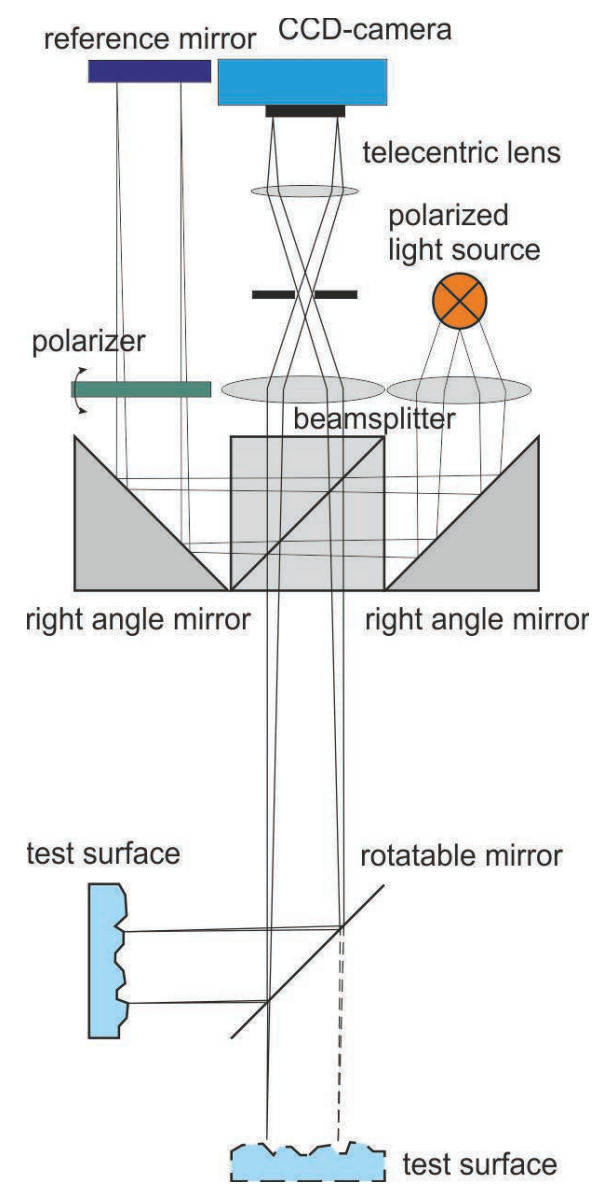

Fig.2. Basic principle of a low coherence interferometer based on a Michelson setup.

A challenge for this measurement system is the size of the sensor head, which has to be suitable to measure the blade's surface, for example, in the small space between two blades in the blisks. For this reason, we use a telecentric lens with a long focus distance to build a compact sensor (see Fig. 3), which is constructed to reduce the size of the sensor head with a rotatable mirror. With the help of the rotatable mirror, sample surfaces can be flexibly scanned parallel or orthogonal to the optical axis. Fig. 3 shows the 3D-CAD-modell of the prototype of the LCl-system.

The low-coherence light is first divided by the beam splitter into two beams to the sample surface and the reference mirror with the same intensity. The reflected light beams from the sample surface and from the reference mirror interfere with each other and are recorded as intensity distribution by the CCD camera. Because the reflected light intensity from the reference mirror is often higher than from the sample surface, the adjustable polarizers in front of the reference mirror allows for the measurement to optimize the contrast of the interference pattern.

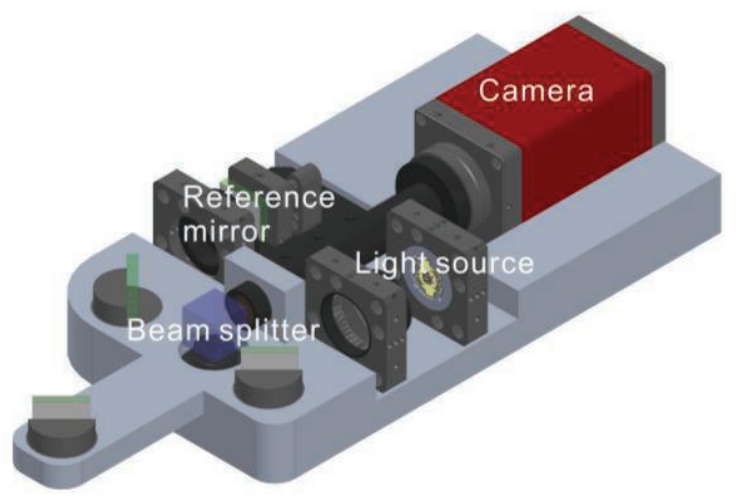

Fig.3. 3D-CAD-modell for the prototype of the LClsystem.

In order to obtain a high vertical resolution, the optical path difference (OPD) between the reference arm and the measurement arm is changed by piezo stage with a nanometer-level step. As the OPD is within the coherence length of the light source, the interference patterns are recorded and stored at each step in a data stack as a layered structure. To synchronize the movement of the piezo stage and the image recording, the measurement procedure is scheduled by an Arduino microcontroller (MEGA 2560), which works as slave device under the host PC. The Arduino receives the information of the measurement sequence and the positioning command for the piezo stage via USB link from the PC. During the measurement procedure, it sends commands to the piezo controller via a RS232 serial port and waits until the movement of the piezo stage is completed. After the positioning, the Arduino sends a trigger-signal to the camera to record an image as soon as it receives the record-ready signal of the camera via monitoring the busy signal. The control flow graph of the $\mathrm{LCl}$-system is shown in Fig. 4 where the movement of the piezo stage and the camera recording are synchronized, so that the scanning time for one step can be reduced to approx. $100 \mathrm{~ms}$. In addition, with the help of the faster data acquisition it is possible to improve the quality of the obtained interference pattern against low-frequency vibrations and drifts during the measuring process [6]. 


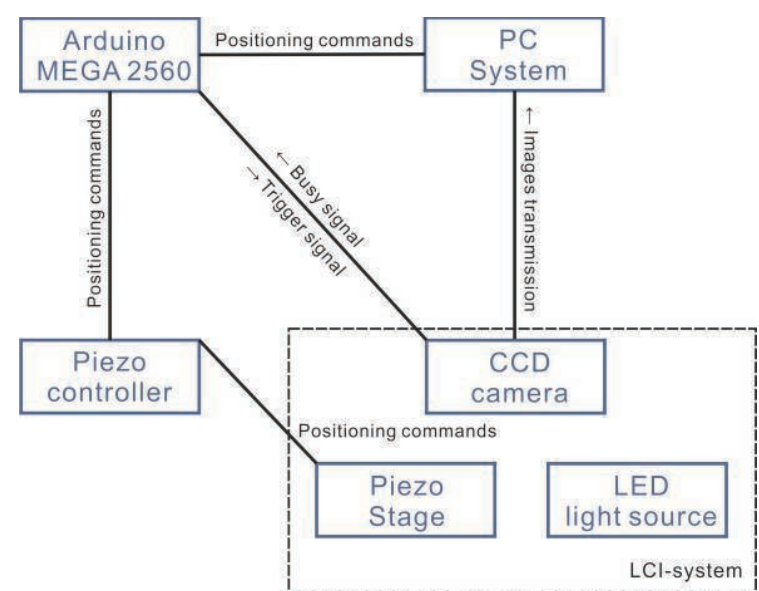

Fig.4. Control flow graph of the LCl-system.

After the data acquisition, the interference correlogram of each pixel with changing OPD can be determined form the stored image stack, as shown in Fig. 5. The correlograms are known as the intensity values of each pixel with 8-bit depth, whose evaluation algorithm is an important part for the accuracy of the LClsystem.

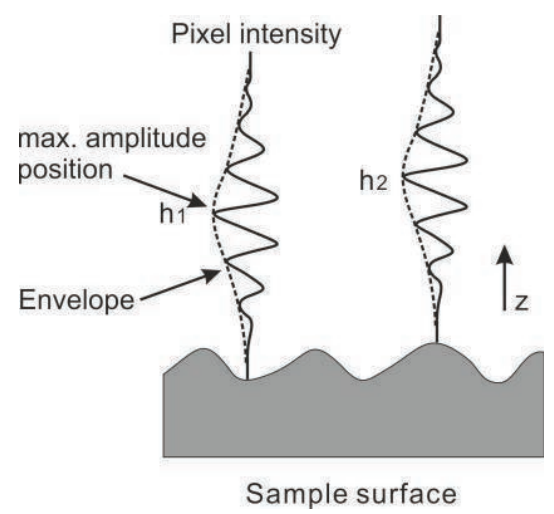

Fig.5. The interference correlogram in $\mathrm{LCl}$ Measurement of the rough surface.
The purpose of the evaluation algorithm is to detect the fringe peak position of the interference correlogram. Firstly, the correlogram is filtered with the help of a bandpass filter, which corresponds to the scan step width and the wavelength. After that, the envelope of the filtered signal is determined with a Hilbert-Transform (HT) algorithm [7], so that the centroid position of the envelope can be converted into the relative surface height of the pixel using the known scan step width of the piezo stage. In fact, using the HT- algorithm and a fine scan step the nanometer-level vertical resolution of the $\mathrm{LCl}$-system can be achieved. Furthermore, the lateral resolution depends on the magnification of the lens and the pixel size of the camera. The in-house developed LCl-system allows a lateral resolution of $1 \mu \mathrm{m}$ with a $4 \mathrm{x}$ telecentric lens.

\section{Experimental results of the LCl-system}

In order to evaluate the performance and the applicability of the LCl-system for measuring the turbine blade, a surface on the leading edge of a worn blade after the removal of the protective layer is measured (see Fig. 6). The microtopographies on the worn blade, for example, dents, nicks or pits can be measured precisely for the analysis of the aerodynamic performance of the turbine blade. However, the depth information of a few pits cannot be measured completely, because in these areas not enough light is reflected back to the camera. Moreover, the unwanted ambient light, which is reflected due to a high surface curvature of the turbine blade, can disturb the accuracy of the measurement result. Therefore, parts of the surface on the convex side of the turbine blade contain measurement noise and some irregularities, as shown in Fig. 6.

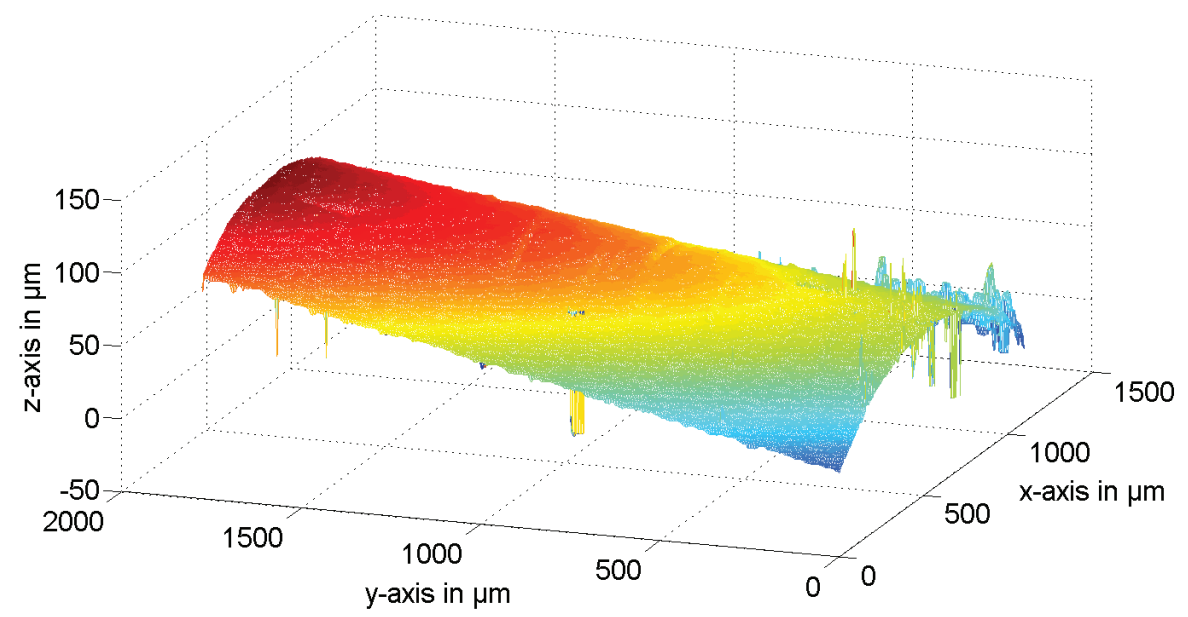

Fig. 6. A measured surface of the leading edge of a worn turbine blade without protective layer. 


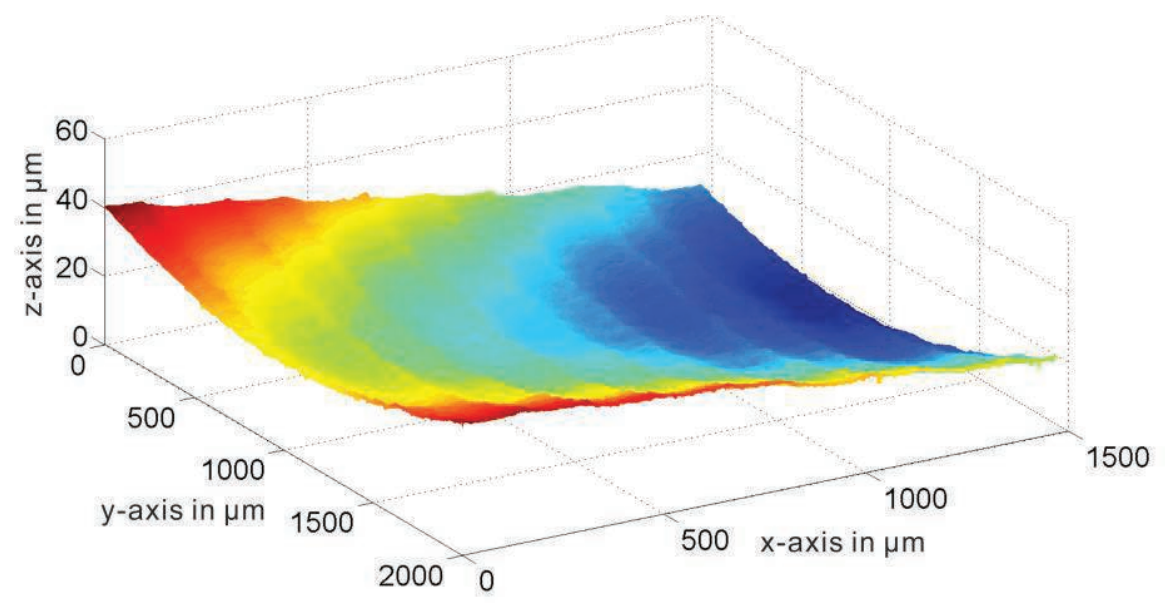

Fig. 7. A measured surface of the pressure-side of the turbine blade, manufactured by a CNC machining.

Fig. 7 shows a measured surface of the pressure-side of the turbine blade, which has been manufactured by a CNC machining system. With the help of the large FOV the shape of the surface can be obtained partly. In production measurement, the waviness and roughness of this surface can be divided from the measurement data using an areal filter (see ISO 4287 [9]). The determined surface microtopographies are especially important for precision machining [10].

\section{Conclusion and Outlook}

The developed LCl-system based on a Michelson interferometer allows a high accuracy surface microtopography measurement and a large FOV using a telecentric lens for measuring curved surfaces, which cannot be measured by a common interferometric microscopy. With a compact design of the sensor head the system is capable of measuring a surface in a small space. However, a part of the measured surface, which obtains very low reflected light due to a high surface curvature, cannot be evaluated for the available height information.

The experimental results show that our LCIsystem can detect the microtopographies on worn turbine blades. And the shape, waviness and roughness of the surface of the new manufactured blades can also be obtained. The determined surface features can possibly be used to detect the surface defects and to improve the precision machining.

In the next step, the compact LCl-system will be installed in an industrial robot based guided system in order to automatically measure the turbine blades. In this situation, the thermal drift and vibrations can disturb the measurement accuracy sensitively. For this application, we will improve the performance of the LCl-system against random drift and vibrations using a high speed camera and develop an effective algorithm to correct the disturbed correlogram. On the other hand, the actual used evaluation algorithms for the large measurement data (high resolution images or even more images for a large vertical range) takes much time in processing. Using parallel computing like multi processing or the general-purpose computing on graphics processing units (GPGPU) [8], it is possible to reduce the computation time for large measurement data.

\section{Acknowledgements}

We would like to thank the German Research Foundation (DFG) for funding this project within the Collaborate Research Center (SFB) 871 "Regeneration of Complex Capital Goods" (http://www.sfb871.de).

\section{References}

[1] F. Engelke, M. Kästner, and E. Reithmeier, Measurement based simulation of microscope deviations for evaluation of stitching algorithms for the extension of Fourier-based alignment. SPIE Optical Metrology. International Society for Optics and Photonics, (2013).

[2] Z. Yibo, M. Kästner, Multiskalenmethode zur dimensionellen Charakterisierung von porösen Oberflächen, DGaO-Proceedings, 115. Jahrestagung in Karlsruhe, ISSN: 1614-8436, (2014).

[3] B. Bhushan, J. C. Wyant, \& C. L. Koliopoulos, Measurement of surface topography of magnetic tapes by Mirau interferometry. Applied Optics, 24(10), 1489-1497, (1985).

[4] F. Hummel, et al. Surface roughness effects on turbine blade aerodynamics. Journal of Turbomachinery 127.3 453-461,(2005):.

[5] J. Ren, S. Sundararajan. Nanoscale surface roughness affects low reynolds number flow: Experiments and modeling. Micro/nano scale surface roughness tailoring and its effect on microfluidic flow 2013;:100. 
[6] M. Melozzi, L. Pezzati, and A. Mazzoni. "Vibration-insensitive interferometer for on-line measurements." Applied optics 34.25 (1995): 5595-5601.

[7] D. A. Zweig, and R. E. Hufnagel, Hilbert transform algorithm for fringe-pattern analysis. San Dieg-DL Tentative. International Society for Optics and Photonics, (1990).

[8] S. Tomczewski, A. Pakula, J. Van Erps, H. Thienpont \& L. Salbut. Low-coherence interferometry with polynomial interpolation on Compute Unified Device Architecture-enabled graphics processing units. Optical Engineering, 52(9), 094105-094105. (2013).

[9] ISO 4287 Geometrical product specification (GPS)_Surface texture: profile method terms, definitions and surface texture parameters. International Organization of Standardization (2000)

[10] A. Taguchi, T. Miyoshi, Y. Takaya \& S. Takahashi. Optical 3D profilometer for in-process measurement of microsurface based on phase retrieval technique. Precision engineering, 28(2), 152-163, (2004). 